\title{
Economic Evaluation of Dulaglutide vs Traditional Therapies: Implications of the Outcomes of the Rewind Study
}

This article was published in the following Dove Press journal: ClinicoEconomics and Outcomes Research

\author{
Enrico Torre' \\ Giacomo M Bruno (iD ${ }^{2}$ \\ Sergio Di Matteo ${ }^{2}$ \\ Chiara Martinotti (iD ${ }^{2}$ \\ Maria Chiara Valentino (iD ${ }^{2}$ \\ Luigi C Bottaro ${ }^{3}$ \\ Giorgio L Colombo iD ${ }^{4}$ \\ 'Endocrinology Diabetology and \\ Metabolic Diseases Unit - ASL3, Genoa, \\ Italy; ${ }^{2}$ S.A.V.E. Studi Analisi Valutazioni \\ Economiche S.r.l., Health Economics \& \\ Outcomes Research, Milan, Italy; \\ ${ }^{3}$ General Direction, ASL3, Genoa, Italy; \\ ${ }^{4}$ Department of Drug Sciences, School of \\ Pharmacy, University of Pavia, Pavia, Italy
}

Correspondence: Giorgio L Colombo Department of Drug Sciences, School of Pharmacy, University of Pavia, Pavia, Italy Email giorgio.colombo@unipv.it
Background: Diabetes represents a relevant public health problem worldwide due to its growing prevalence and socioeconomic burden, principally due to the development of macrovascular and microvascular complications as well as to the continuous launch of new and even more expensive drugs. The aim of our study is to evaluate the economic impact of dulaglutide, a weekly GLP-1 receptor agonist, on the treatment of diabetic patients as an alternative to both high dose sulphonylureas and insulin basalization at the failure of oral therapies alone. We carried out a cost-effectiveness analysis developed considering the economic implications of recent clinical studies regarding cardiovascular risk drug effects and especially of REWIND studies outcomes, focusing on the impact of weight changes on HRQoL.

Material and Method: In our analysis, we have applied the cost-utility technique to the above reported clinical outcomes and compared the global costs of dulaglutide versus sulfonylurea or basal insulin, all in add-on with metformin. We have chosen gliclazide, as a sulfonylurea and Abasaglar $^{\circledR}$, the less expensive among basal insulin analogues. Abasaglar was titrated to $20 \mathrm{IU}$, corresponding to the mean dosage used in the treatment of type II diabetic patients. The model aims to estimate total direct costs related to the above-reported treatments and find out the real gap in costs between dulaglutide, the apparently cheaper gliclazide and basal insulin glargine (IGlargine) based on the Italian National Healthcare System (INHS).

Results: The total cost of dulaglutide has resulted in $€ 859.66$ higher than gliclazide $(€ 1,579.73$ vs $€ 720.07)$ and basal insulin, although less significantly, reporting a difference of $€ 396.54$ ( $€ 1,579.73$ vs $1,183.19$ ). Except for the purchase cost, dulaglutide has reported reduced costs compared to insulin IGlargine and gliclazide. Dulaglutide showed lower selfmonitoring blood glucose and hypoglycaemia costs, a significant reduction in costs related to cardiovascular complications, as well as savings in costs in other drugs. Dulaglutide can be considered a cost-effective antidiabetic therapy, due to the positive impact on the quality of life induced by weight reduction, despite the higher annual cost per patient, mainly influenced by drug purchase cost.

Discussion and Conclusion: In this cost-utility analysis, dulaglutide has shown to be a cost-effective treatment option from the Italian healthcare system perspective as add-on therapy to metformin in patients with inadequately controlled type 2 diabetes mellitus. Study findings can provide stakeholders valuable evidence to support the adoption of this costeffective second- or third-line therapy compared to gliclazide or basal insulin glargine. Dulaglutide cost-effectiveness has been particularly evident in the comparison with basal insulin glargine, indicating that, in patients who have treatment indication, this therapy may be preferred to basalization avoiding related complications and costs.

Keywords: dulaglutide, cost utility analysis, diabetes type II 


\section{Introduction}

The aim of our study is to evaluate the economic impact of dulaglutide, a weekly GLP-1 receptor agonist, on the treatment of diabetic patients as an alternative to both high dose sulphonylureas and insulin basalization at the failure of oral therapies alone.

Diabetes represents a relevant public health problem worldwide due to its growing prevalence and socioeconomic burden, principally due to the development of macrovascular and microvascular complications as well as to the continuous launch of new and even more expensive drugs.

All antidiabetic agents marketed from 2000 onwards guarantee a very low hypoglycemic risk.

They have been tested to ensure cardiovascular safety and many of them even showed a reduction in cardiovascular risk. Despite the clinical benefits of these therapies, attention to cost containment may limit their use. In Italy, more than 3.2 million people reported to suffer from diabetes, $5.3 \%$ of the total population. ${ }^{1}$ Currently, $67 \%$ of the patients are treated with oral hypoglycemic agents (OHA), $10 \%$ of them with a combination of insulin and OHA, and $11 \%$ with insulin alone. ${ }^{2} 20.3 \%$ of patients treated with OHA is still treated with sulfonylureas. ${ }^{3}$ Moreover, $18.8 \%$ of diabetic patients in our country have HbAlc levels higher than $64 \mathrm{mmol} / \mathrm{mol}^{3}$ Patients with suboptimal metabolic control present an increased risk of developing chronic and acute microvascular and macrovascular complications as well as severe hypoglycemia, both affecting patients' survival, quality of life and costs. No doubts that tight glycemic control reduces the development of diabetic complications such as the long-term costs related to the disease. ${ }^{4-6}$ On the other side, tight glycemic control is often neglected in order to avoid treatment-related hypoglycemia. ${ }^{7}$

From Italian NHS perspective, no economic data are currently available for GLP-1 receptor agonists, a class of drugs that can represent a valid option failure to monotherapy with metformin, while some studies on the economic value of DPP4 inhibitors and SGLT2 inhibitors have been published. ${ }^{8,9}$ GLP-1 receptor agonists due to their hypoglycemic power represent a valid alternative to basal insulinization failure to oral agents.

GLP-1 (Glucagon-like peptide-1) is the hormone responsible for incretin effect, which consists in a response to a higher insulin release due to relevant glucose levels after a meal. Type 2 diabetic patients show an attenuated incretin effect, probably because of reduced levels of active GLP-1. ${ }^{10}$ The main GLP-1 effects include an improvement of beta-cell function, the suppression of glucagon release and of the hepatic glucose output. It also decreases the rate of gastric emptying and acid secretion, thereby reducing appetite and contributing to weight loss. ${ }^{11}$

Dulaglutide, approved for the treatment of hyperglycemia in people with diabetes in many countries, is administered subcutaneously at weekly doses of 0.75 and $1.5 \mathrm{mg}$ (the latter more frequently used) and safely reduces glucose concentrations, blood pressure, weight and albuminuria. ${ }^{12-14}$ Dulaglutide has recently showed cardiovascular benefits as well. ${ }^{15}$ The drug, administered at the dosage of both 0.75 and $1.5 \mathrm{mg}$ per week has shown to reduce $\mathrm{HbAlc}$ of $-1.1 \%-12 \mathrm{mmol} / \mathrm{mol}(\mathrm{p}<0.0001)$ in a comparative study with titrated basal insulin analogue (glargine) which demonstrated the same hypoglycemic power $(-1.0 \% ; \mathrm{p}<0.0001)$, based on a similar proportion among all participants in reaching HbAlc targets lower than $8 \%-64 \mathrm{mmol} / \mathrm{mol}$ and lower than $7 \%-53 \mathrm{mmol} / \mathrm{mol}$, at both 26 and 52 weeks. ${ }^{14}$ Both dulaglutide doses were non-inferior to insulin glargine with a $0-4 \%$ noninferiority margin (one-sided $\mathrm{p} \leq 0.0001$ ); as a further outcome of this study, bodyweight decreased at 52 weeks with both dulaglutide doses $(-2.5 \mathrm{~kg}$ for $1.5 \mathrm{mg}$, $\mathrm{p}<0.001 ;-1.5 \mathrm{~kg}$ for $0.75 \mathrm{mg}, \mathrm{p}<0.0001)$ and increased for glargine $(+1.5 \mathrm{~kg}, \mathrm{p}=0.003) .{ }^{14}$ Dulaglutide has also demonstrated to be superior to sulphonylureas in a 24week randomized vs placebo study, achieving targets of $\mathrm{HbAlc}<7 \%$ in $55.3 \%(9.4 \% \leq 6.5 \% ; \mathrm{p}<0.001)$ in patients which were not at target with glimepiride alone. ${ }^{16}$

Compared to insulin and sulphonylureas, dulaglutide shows a very low incidence of hypoglycemia; 0.89 events/ patient/year for $1.5 \mathrm{mg}$ dosage ( 0.47 for $0.75 \mathrm{mg}$ ) slightly more than the 0.29 events/patient/year detected for metformin, with no reporting of severe ones. ${ }^{17}$ Regarding cardiovascular risk, several studies on different GLP-1 agonists demonstrated a reduction in major adverse cardiac events (MACE) ${ }^{18}$ but all of them based on populations selected by criteria of higher prevalence of cardiovascular history. REWIND ${ }^{15}$ dulaglutide trial is otherwise the first study on a GLP-1 agonist demonstrating a reduction in MACE composite on a population comparable to real life and over a period of more than 5 years. REWIND was a multicentre, randomized double-blind, placebo-controlled trial done at 371 sites in 24 countries. Patients presented established or newly detected type 2 diabetes whose $\mathrm{HbAlc}$ was $9.5 \%-80 \mathrm{mmol} / \mathrm{mol}$ or less 
on stable doses of up to two oral glucose-lowering drugs with or without basal insulin therapy. After 3-week run-in period patients were randomly assigned to dulaglutide or placebo. The primary endpoint of the study was the first occurrence of any component of the composite outcome, which included non-fatal myocardial infarction, non-fatal stroke, and death from cardiovascular or unknown causes. The primary composite outcome occurred in 594 (12.0\%) participants (2.4 per 100 persons-years) assigned to dulaglutide and 663 participants $(13.4 \%$; 2.7 per 100 personsyear) assigned to placebo (HR 0.88, 95\% CI 0.79-0.99; $\mathrm{p}=0.026$ ). Cardiovascular disease represents the most important complication of diabetes both for clinical, social aspects and costs. Reducing the economic burden of cardiovascular disease in diabetic patients is, therefore, an important goal of all antidiabetic medications, and the new therapies available can play an important role. ${ }^{19}$

Recently, it has been published an interesting US retrospective cohort study ${ }^{20}$ based on national administrative claims data compared to the cardiovascular effectiveness of the next antidiabetic medication class started after metformin alone or no prior antidiabetic medication: DPP4 inhibitors, GLP-1 receptor agonists, SGLT-2 inhibitors, thiazolidinediones (TZDs), basal insulin, or sulfonylureas. In our opinion, this study, conducted on a population of 132,737 adult diabetic patients, can represent a good support to the valorization of dulaglutide. The composite primary outcome included hospitalization for one of the following cardiovascular conditions: congestive heart failure, stroke, ischemic heart disease, and peripheral artery disease; moreover, all the considered events were also examined individually as secondary outcomes. Statistical analysis was developed considering DPP4 inhibitors as a benchmark treatment and it demonstrated that the starting treatment with GLP-1 receptor agonists was associated with a lower incidence of composite cardiovascular events (HR 0.78; 95\% CI 0.63-0.96), as subsequently confirmed also by the REWIND study, whereas SGLT2i and TZDs resulted not statistically different from the benchmark drug. In this study, the risk of MACE was 36\% higher in the sulfonylureas group (HR 1.36; 95\% CI 1.23-1.49) and more than two times higher in the basal insulin group (HR 2.03 ; 95\% CI 1.81-2.27) than in the DPP-4 inhibitor group. Increased relative cardiovascular risk associated with the use of sulfonylureas or basal insulin was observed across all individual cardiovascular outcomes. Treatment with GLP-1 receptor agonists was associated with a significant reduction in stroke risk (HR 0.65; 95\% CI
0.44-0.97), as subsequently confirmed by the same outcome in REWIND study. Hence, dulaglutide not only has a reduced risk of cardiovascular complications (stroke) but it also can avoid the additional cardiovascular events generated by sulfonylureas or insulin. The last consideration is relevant for any economic evaluation of antidiabetic agents. The effect on body weight shown by dulaglutide (more evident versus insulin with a $4 \mathrm{~kg}$ delta) ${ }^{14}$ is also to be noted, even if its impact on health costs is more difficult to evaluate, requiring an analysis based on Quality Adjusted Life Years (QALY) measure. To conclude, dulaglutide provides equal or better $\mathrm{HbAlc}$ levels than the ones obtainable with maximum doses of sulfonylurea (ie gliclazide) and can also represent a valid alternative to basal insulin. ${ }^{14}$ This outcome is achieved by exposing the patient to lower $\mathrm{CV}$ and mortality risk, lower hypoglycemic risk, lower need for blood glucose self-monitoring tests, lower need of hospitalization for HF, and, last but not least, a reduction of weight and general improvement in the quality of life. All these aspects are relevant from the point of view of the costs of treatment. Specifically, given the remarkable drug effect on weight loss, we have decided to estimate what the impact of this reduction on patient's quality of life may be. To do it, we have developed a cost-utility analysis, a type of cost-effectiveness study, in order to show the economic value of dulaglutide treatment. Cost-effectiveness analysis describes the cost per unit health outcome obtained with an experimental intervention versus usual care. ${ }^{21}$ It provides a measure of the value for the money spent and may be used to guide resource allocation. In cost-utility analysis, health outcomes are measured by quality-adjusted life-years (QALYs), a metric that adjusts the length of life for quality-of-life. Quality-of-life is assessed with health utility scores, measures that reflect the general population's preference for specific health states. Health utility scores fall on a continuum between 1.0 and 0 , where 1.0 represents perfect health and 0 represents death. QALYs are calculated as the sum of the product of the number of years of life and the quality-of-life in each of those years. The goal of an experimental intervention is to maximize the number of QALYs-gained for the money spent related to usual care. The cost-effectiveness analysis was developed considering the economic implications of recent clinical studies regarding cardiovascular risk drug effects and especially of REWIND studies outcomes, focusing on the impact of weight changes on HRQoL. 


\section{Materials and Methods}

We have developed a cost-utility analysis based on the current clinical evidence previously reported. We have compared the global costs of dulaglutide versus sulfonylurea or basal insulin, all in add-on with Metformin. We have chosen gliclazide as sulfonylurea and the less expensive among basal insulin analogues, that is insulin glargine, this one titrated to $20 \mathrm{IU}$, corresponding to the mean dosage used in the treatment of type II diabetic patients. $^{22,23}$

Our model aims to estimate the total direct costs related to the above-reported treatments and to find out the real gap in costs between dulaglutide and the apparently cheaper gliclazide and insulin glargine. In order to reflect the perspective of Italian National Healthcare System INHS, direct costs related to treatment and management of main diabetes complications have been included. In this analysis, we have considered as direct costs all the resources adopted to provide healthcare services as well as those used to counteract the possible adverse effects of the therapies used. Finally, we have developed a cost-utility analysis model using the Incremental Cost-Effectiveness Ratio (ICER). Utility scores have been measured based on patient's perception of weight changes. Indirect costs have not been considered in the main scenario, however, to extend the analysis perspective and keep the way open for subsequent evaluations, the effect on total costs, also considering the indirect costs, has been estimated, as expressed in the discussions.

\section{Model Description}

Our model, developed in Microsoft Excel, ${ }^{\circledR}$ has compared the costs of the above mentioned therapeutic options, including costs of therapy management and side effects, both negative and positive, while the costs which do not vary within the different scenarios, such as costs of metformin and outpatients costs, have not been included in the calculation. We also have decided to not include the costs related to peripheral artery disease due to the difficulty of evaluation.

The following cost items have been adopted to evaluate total direct cost:

- The cost of the drug (including needles if injectable)

- The cost of glycemic self-monitoring

- Direct costs related to hypoglycemic events

- Direct costs related to stroke
- Direct costs related to nonfatal ischemic heart disease (IHD)

- Direct costs related to congestive heart failure (HF)

- Costs and gains related to the consumption or spare of other drugs

The model, based on a one-year time mark, has been developed referring to Italian NHS and data have been inputted by reference to the scientific literature, using Italian evaluation of costs whenever possible and referring to internationally acknowledged data when it was not possible otherwise. We considered only direct costs to define the NHS perspective. Drug costs have been calculated considering NHS real dispensation costs, which correspond to list prices for generic products (such as gliclazide) and to ex-factory rebated price for the other drugs considered in our model (insulin included). Benefits related to new antidiabetic drugs also include the positive effect on weight reduction that allows improvements in the patient's quality of life as demonstrated in recent studies. In order to assess the impact of changes in T2DM patient's body weight on health-related quality-of-life, we have used utilities data from the literature, ${ }^{24}$ and we have calculated utility values for weight changes related to dulaglutide, gliclazide and insulin glargine. Finally, we compared the difference between total costs and utility costs related to weight changes, in order to assess the overall benefit for patient taking dulaglutide through a cost-utility analysis. The results of cost-utility analysis have been expressed as an incremental cost-effectiveness ratio (ICER), which has been calculated as the difference in the cost of the two therapies, divided by the difference in the QALYs. This ratio of Cost/QALY has been then compared with a threshold ICER (willingness to pay). Willingness to pay differs from country to country. Although no officially established value is available for Italy, it is worth noting that recent guidelines by the Italian Health Economics Association (AIES) recommend that a threshold of $€ 25$,000-40,000 be adopted. ${ }^{25}$ To assess the robustness of results, a one-way sensitivity analysis was performed by changing the main parameters of efficacy and costs by $\pm 20 \%$. Moreover, since the drug purchase cost is a variable that significantly influences the overall treatment cost, we have developed some simulation scenarios taking into account the impact on drug price exercised by any hidden discounts applied to the ex-factory price, to provide an economic evaluation as realistic as possible. Indeed, the purchase costs of drugs are usually subject to hidden 
discounts, except for generic products, the costs of which are included in a transparency list. Finally, in the discussions, we have included an estimate of the inclusion of indirect costs in the model.

\section{Cost of Drugs}

Reporting drug costs we have considered the price that can more appropriately reflect actual spending for the Italian NHS (ASL). More specifically, we have taken into consideration the price based on the reimbursement band provided and the possible adherence to the payback.

For generic products, public list prices have been adopted, while for the other ones, the costs of drugs have been deducted from ex-factory prices (with $-5-5 \%$ mandatory rebates if not subject to payback or with $-5 \%$ if it is applicable). ${ }^{26}$ We have calculated the annual direct cost per patient of each drug using the following dosages: dulaglutide: $1.5 \mathrm{mg}$ once a week, gliclazide: $60 \mathrm{mg}$ twice a day, insulin glargine $20 \mathrm{IU}$ per day, as reported above. Data adopted to calculate the costs of pharmacological treatment (included the costs related to needles for insulin therapy) are shown in Table 1.

\section{Cost of Glycemic Self-Monitoring}

To evaluate the costs of glycemic self-monitoring we have considered the number of glycemic controls per week, for the recommended frequency of checks related to therapy we have referred to national scientific societies (AMD-SID) guidelines, ${ }^{27}$ reporting a mean value extrapolated from these indications. In Italy, the cost of stripes differs from region to region, with an average price per unit of about $€$ 0.55 . In our model, we have adopted the last price suggested by CONSIP (Italian public procurement office), which amounts to $€ 0.35$. We must also consider that, even if prices resulting in the latest public tenders are getting lower and lower, distribution costs remain unchanged so determining an incompressibility of real costs, ${ }^{28}$ not considering that new technologies in glycemic monitoring (i.e. flash monitoring portable devices) are spreading and this will probably bring up average costs again, at least for insulin-treated patients. In our model we have also considered the cost of blood sampling, amounting to $€ 0.0145$ per lancet in accordance with acquisition cost in Liguria Region, chosen as a benchmark. ${ }^{29}$

The input data adopted to calculate the costs of glycemic self-monitoring are shown in Table 2.

\section{Hypoglycemic Events: Rates and Costs}

A severe hypoglycemic event is defined as an episode with symptoms of low blood glucose levels during which the patient requires assistance from another person, mainly through hospitalization; when the patient can solve the problem by itself, the event is defined as moderate. To estimate the hypoglycemia cost we have referred to the incidence of hypoglycemic events reported in international literature for patients with type 2 diabetes. ${ }^{30,31}$ Regarding the evaluation of direct costs, we have considered only severe and moderate hypoglycemia, not including mild ones because of their negligible economic impact. We have inputted direct mean costs of severe hypoglycemic events, valued equal to $€ 1911$, referring to an Italian study, ${ }^{32}$ whereas for moderate ones we have adopted a large Swedish study which reported data about it, already cited above. ${ }^{30}$ For each treatment, we have considered direct costs per patient/year combining the mean cost per event with the incidence of events related to each drug. According to available literature, dulaglutide incidence of severe hypoglycemia is close to zero, whereas the highest values are reported for insulin treatment (0.1180) and gliclazide (0.0116). ${ }^{33}$ Data on rates and costs of hypoglycemic events are reported in Table 3.

\section{Cardiovascular Complications: Rates and Costs}

Data concerning the effect of hypoglycemic therapies on cardiovascular risk have been extrapolated from the recent studies cited above. ${ }^{15,20}$ Risk values (Hazard Ratio, HR)

Table I Pharmacological Treatment Costs: Drug Cost

\begin{tabular}{|l|l|l|l|l|l|l|}
\hline \multirow{2}{*}{ API } & Product & Content & Dose per & Posology & Public & Ex-factory \\
\cline { 2 - 7 } & Specifications & Per Package & Unit (mg/UI) & (Units Die) & List Price & Price \\
\hline $\begin{array}{l}\text { Dulaglutide I.5 mg } \\
\text { IGla-100 bios. }\end{array}$ & Trulicity $^{\circledR}$ & 4 pens $0.5 \mathrm{~mL}$ & $1.5 \mathrm{mg}$ & 0.14 & $€ 161.56$ & $€ 97.89$ \\
Gliclazide $60 \mathrm{mg}$ & Abasaglar & Generic & 5 pens $3 \mathrm{~mL}$ & $300 \mathrm{U}$ & 20 & $\begin{array}{l}€ 60.69 \\
€ 5.37\end{array}$ \\
\hline Needle BD 5mm & & 30 tab. & $60 \mathrm{mg}$ & 2 & $€ 2.77$ \\
\hline
\end{tabular}

Abbreviations: API, active pharmaceutical ingredient; IGla- 100 bios, Insulin Glargine $100 \mathrm{U} / \mathrm{mL}$ Biosimilar; tab, tablet; UI, units of insulin. 
Table 2 Treatment Costs: Glycemic Self-Monitoring Costs

\begin{tabular}{|l|l|l|l|}
\hline \multirow{2}{*}{ API } & \multirow{2}{*}{ Weekly SBGM } & Lancet & Strip \\
\cline { 3 - 4 } & & Cost & Cost \\
\hline Dulaglutide & $\mathrm{I}$ & $€ 0.0145$ & $€ 0.35$ \\
IGla-I00 bios. & 8 & $€ 0.0145$ & $€ 0.35$ \\
Gliclazide & 4 & $€ 0.0145$ & $€ 0.35$ \\
\hline
\end{tabular}

Abbreviations: API, active pharmaceutical ingredient; SBGM, self-blood glucose monitoring; IGla-100 bios, Insulin Glargine $100 \mathrm{U} / \mathrm{mL}$ Biosimilar.

Table 3 Hypoglycemic Events: Rates and Costs

\begin{tabular}{|l|l|l|}
\hline \multirow{2}{*}{ API } & \multicolumn{2}{|l|}{ Hypoglycemic Event Rates (\%) } \\
\cline { 2 - 3 } & Moderate & Severe \\
\hline Dulaglutide & $7.00 \%$ & $0.19 \%$ \\
IGla-100 bios. & $8.60 \%$ & $11.80 \%$ \\
Gliclazide & $22.00 \%$ & $1.16 \%$ \\
Direct cost per event & $€ 334.7$ & $€ 1,911.00$ \\
\hline
\end{tabular}

Abbreviatons: API, active pharmaceutical ingredient; IGla-100 bios, Insulin Glargine $100 \mathrm{U} / \mathrm{mL}$ Biosimilar.

for IHD, stroke and hospitalization for heart failure associated with the consumption of dulaglutide, basal insulin and sulphanilureas have been considered. For dulaglutide, if statistically significant, as in the case of HR for stroke, specific outcomes have been adopted from REWIND study results, if the effect on risk has not been statistically significant, neutral impact has been considered and frequencies of events in reference population have been adopted. Direct costs for IHD, stroke and hospitalization for heart failure have been derived from national data, as below reported.

\section{Ischemic Heart Disease (IHD)}

Whereas REWIND study has demonstrated a significant reduction in a composite cardiovascular outcome (nonfatal myocardial infarction, non-fatal stroke, or death from cardiovascular causes) related to dulaglutide use, ${ }^{15}$ we have not included this data in our analysis because of the difficulty in quantifying it, and regarding each component of the composite cardiovascular outcome, we have adopted it only when statistically significant. Then, since the effect of the therapy on the incidence of IHD has not been significant, the incidence in the reference target population has been adopted in the calculation. On the other hand, we have included in our model the major incidence of non-fatal IHD reported in the above reported US retrospective cohort study ${ }^{20}$ for both Sulfonylureas and insulin (HR 1.35; 95\% CI 1.16-1.57 and HR 1.92; 95\% CI
1.59-2.32, respectively). To quantify the direct costs of IHD, we have utilized the costs described in an Italian study based on "Osservatorio ARNO" population. ${ }^{34} \mathrm{We}$ have started from the reported incidence of IHD and we have applied the $8.4 \%$ incremental correction coefficient defined for diabetic patients versus general population in the same study. Event rates and cost per event for IHD are shown in Table 4.

\section{Stroke}

Among secondary outcomes of this REWIND study, the incidence of non-fatal stroke has resulted in $0.76(0.61-$ $0.95 ; \mathrm{p}=0.017)$, in line with the above reported US retrospective cohort study (HR $0.65 ; 95 \% \mathrm{CI} 0.44-0.97) .{ }^{20} \mathrm{We}$ chose the first HR (0.76) instead of the better second $(0.65)$ as it is a specific outcome for dulaglutide.

As we have done for IHD, we have included in our model the major incidence of non-fatal stroke reported in same US retrospective cohort study, ${ }^{20}$ for both sulfonylureas and insulin glargine (HR 1.28; 95\% CI 1.08-1.52 and HR 1.77; 95\% CI 1.44-2.19, respectively). We have therefore included in our economic model the direct costs of stroke described in the above reported Italian ARNO population study. ${ }^{32}$ Event rates and cost per event for stroke are shown in Table 4.

\section{Heat Failure (HF)}

Heart failure is highly prevalent in diabetic patients, occurring in more than $20 \%$ of those aged over 65 years, so that HF amounts to about $0.51 / 100$ person-years. ${ }^{35}$ For dulaglutide, we have used this data, while also for HF we included in our model the major incidence of congestive heart failure

Table 4 Cardiovascular Diabetes Complications: Event Rates and Costs

\begin{tabular}{|c|c|c|c|}
\hline & IHD & Stroke & HF \\
\hline Rates in Target Population & $0.97 \%$ & $0.59 \%$ & $0.51 \%$ \\
\hline API & \multicolumn{3}{|l|}{ HR } \\
\hline Dulaglutide & 1 & 0.76 & I \\
\hline IGla-I00 bios. & 1.92 & 1.77 & 2.33 \\
\hline \multirow[t]{3}{*}{ Gliclazide } & 1.35 & 1.28 & 1.47 \\
\hline & IHD & Stroke & HF \\
\hline & \multicolumn{3}{|c|}{ Event Costs } \\
\hline Direct cost per event & $€ 15,949$ & $€ 10,237$ & $€ I I, 000$ \\
\hline
\end{tabular}

Abbreviations: API, active pharmaceutical ingredient; IHD, Ischemic Heart Disease; HF, Heart failure. 
reported in the US retrospective cohort study, ${ }^{20}$ always for both sulfonylureas and insulin (HR 1.47; 95\% CI 1.23-1.75 and HR 2.33; 95\% CI 1.90-2.87, respectively). We then included in our economic model the direct costs of HF related to the use of sulfonylureas or basal insulin. In our country, health spending related to HF amounts to $€ 11,100$ per person/ year considering only direct costs. ${ }^{36}$ Event rates and cost per event for HF are shown in Table 4. Table 4 shows the risk of IHD, stroke and hospitalization for heart failure occurrence, respectively, for dulaglutide, insulin glargine and gliclazide. Specifically, the hazard ratio is reported for each drug, highlighting the different impact of therapies on events over a year, compared to the frequencies of occurrence in the reference population $(0.97 \%$ for IHD, $0.59 \%$ for stroke, and $0.51 \%$ for HHF). Moreover, direct cost per event is reported in Table 4.

\section{Costs Related to the Consumption of Other Drugs}

Dulaglutide, like the other GLP-1 agonists, reduces also systolic blood pressure in patients with type 2 diabetes, including those receiving concomitant antihypertensive medication; this reduction amounts to an average of -3.1 $\mathrm{mmHg}$ and results statistically significant $(\mathrm{p}=0.001){ }^{37}$

In order to economically quantify this benefit, we have applied the mean cost of a generic antihypertensive medication (ie doxazosine/amlodipine), which amounts to about $€ 0.228$ per day, to the prevalence of hypertension in Italian diabetic population $(58.6 \%)$, as reported in our AMD (National Diabetologist Association) annals. ${ }^{38}$ The use of dulaglutide, therefore, allows a saving of $€ 48.8$ per patient on annual antihypertensive therapy cost.

\section{Change in Weight and Utilities Calculation}

We developed a cost-analysis regarding different antidiabetic therapies. However, we also aim to extend our evaluation on patients' quality of life.

In particular, we have considered the effects of body weight changes. It is known that some classes of antidiabetic drugs involve an increase in the patient's weight, among these surely the sulfonylureas while, on the other hand, treatment with GLP-1 agonist and dulaglutide among these is associated with a significant reduction in weight. ${ }^{14,39,40}$

Moreover, aiming to include in our evaluation the impact of body weight on patients' perceptions of quality of life, we performed a literature analysis aimed at identifying the weight variations associated with compared drugs, dulaglutide, gliclazide and insulin glargine, considering a one-year time period. It has been shown, on one hand, a weight increase of $1.5 \mathrm{~kg}$ in patients treated with gliclazide or insulin glargine on the other one a weight reduction of $2.5 \mathrm{~kg}$ in patients treated with dulaglutide. ${ }^{14,41}$ Hence, we applied these differences in weight to utility measures calculated in a Swedish study, which shows for loss and gain of $2 \mathrm{~kg}$ weight utility values of 0.92 and 0.88 , respectively. ${ }^{24}$ By combining a weight variation (4 $\mathrm{kg}$ delta reported in Ridderstråle's study ${ }^{24}$ and utility values, we have calculated the utility value related to $1 \mathrm{~kg}$ variation, such as to be easily associated with the specific weight changes highlighted in the literature for a year of treatment with drugs compared in our analysis, obtaining denominators for the cost-utility analysis.

\section{Sensitivity Analysis}

In order to assess the robustness of our analysis, a one-way sensitivity analysis has been carried out varying by $\pm 20 \%$ of the main input parameters in the model, namely, cost data and efficacy data (complications and side effects rates and utilities values). Furthermore, since the purchase costs of drugs are usually subject to hidden discounts, except for generic products, the sensitivity of the results has been tested considering the addition of a percent discount. Specifically, in order to reproduce an economic evaluation as close as possible to the real direct cost paid by hospitals, we have tested the effects of increasing discount rates applied to ex-factory price $(40 \%, 45 \%, 50 \%)$.

\section{Results}

We have calculated the total annual cost per patient related to dulaglutide versus gliclazide and insulin glargine for T2D patients not achieving glycemic control on metformin. Total treatment costs have been obtained by adding direct annual cost of drug, needles, glycemic selfmonitoring, hypoglycaemic events, cardiovascular complications, and effect on other drugs consumption. Individual cost items making up total treatment annual costs per patient for each therapy are shown in Figure 1 and Table 5.

The total cost of dulaglutide has resulted in $€ 859.66$ higher than gliclazide ( $€ 1,579.73$ vs $€ 720.07)$, and greater compared to basal insulin, although less significantly, reporting a difference of $€ 396.54 \quad(€ 1,579.73$ vs 1,183.19). However, after breaking down the final cost in the individual items, in Figure 1 we clearly note that the high total cost of dulaglutide is mainly determined by 


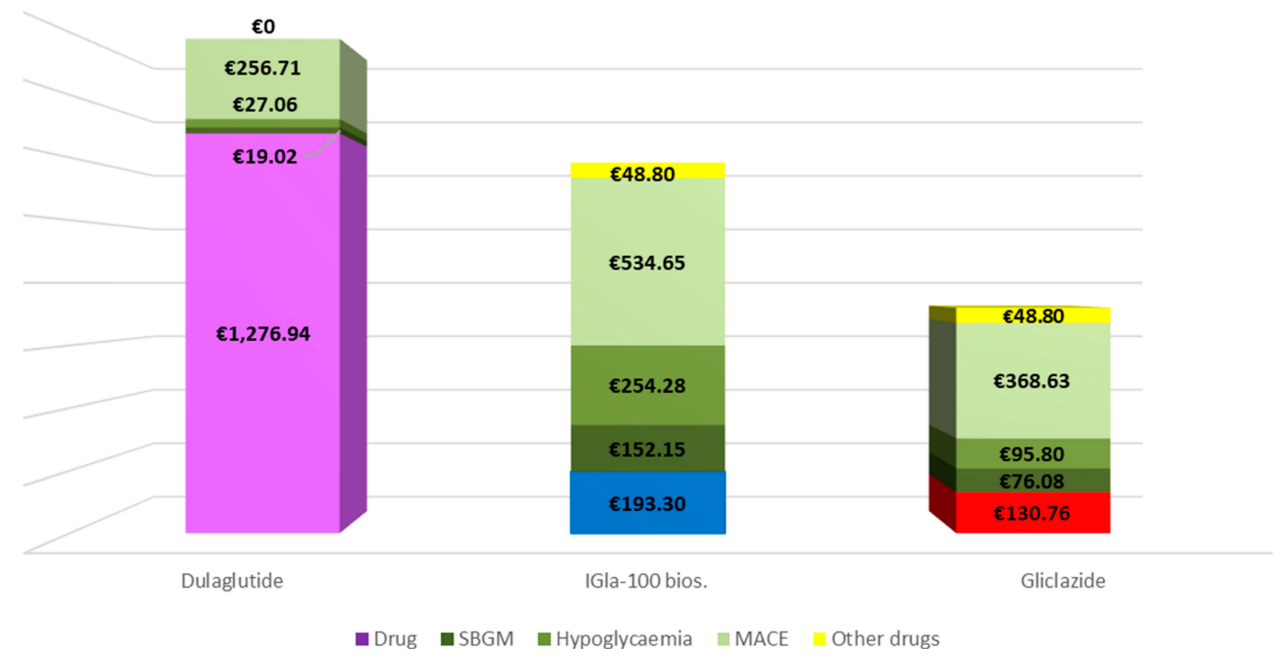

Figure I Direct costs: individual items for each treatment.

purchase cost, that is about nine times higher than that of gliclazide, the drug of consolidated use, now available in generic form. As far as the comparison with insulin glargine is concerned, total annual costs report a less significant difference although in this case, the difference in annual purchase cost is considerable (more than 6 times higher), due to the increase in cost given for other items for insulin glargine, as monitoring costs and cardiovascular complications costs. Globally, except for the purchase cost, dulaglutide has shown reduced costs compared to insulin glargine and gliclazide. It has involved lower selfmonitoring blood glucose costs, lower costs related to hypoglycaemia, a significant reduction in costs related to cardiovascular complications, as well as saving in other drugs costs, Table 5 and Figure 1.

Based on utility values for $2 \mathrm{~kg}$ weight gain or $2 \mathrm{~kg}$ weight loss, ${ }^{24}$ the utility for $1 \mathrm{~kg}$ variation has been obtained equal to 0.010 . Multiplying this value by the weight variation associated with 1 year of treatment with dulaglutide, insulin glargine or gliclazide (as per literature sources), the utility measurements for these drugs have resulted in 0.025 for dulaglutide and -0.015 for the last two ones.
The difference between total costs of dulaglutide and, respectively, of insulin glargine and gliclazide have been divided by the difference between the utility measurements, obtaining positive incremental cost-effectiveness ratio in our analysis, Table 6 . The additional cost per quality-adjusted life-year (QALY) gained has been $€ 9913$ in comparison with insulin glargine and $€ 21,492$ when compared with gliclazide. The incremental cost-effectiveness ratios we obtained have fully fallen within acceptable willingness to pay threshold in Italy. Consequently, dulaglutide can be considered a cost-effective antidiabetic therapy, due to the positive effect on the quality of life induced by weight reduction, despite the higher annual cost per patient, mainly influenced by drug purchase cost.

\section{Sensitivity Analyses}

The sensitivity analysis has shown the robustness of the results. Actually, the variation of cost and effectiveness data $( \pm 20 \%)$ has not resulted in significant deviations from base case findings, always keeping ICERs well within the acceptable threshold, Table 7 and Figure 2. Furthermore, we have carried out a sensitivity analysis assuming feasible scenarios

Table 5 Total Annual Direct Cost of Treatments

\begin{tabular}{|c|c|c|c|c|c|c|c|c|}
\hline \multirow[t]{2}{*}{ API } & \multirow[t]{2}{*}{ Drug } & \multirow[t]{2}{*}{ SMBG } & \multirow[t]{2}{*}{ Hypoglycaemia } & \multicolumn{3}{|l|}{ MACE } & \multirow[t]{2}{*}{ Other Drugs } & \multirow[t]{2}{*}{ Total Cost } \\
\hline & & & & IHD & Stroke & HF & & \\
\hline Dulaglutide & $€ I, 276.94$ & $€ 19.02$ & $€ 27.06$ & $€|54.7|$ & $€ 45.90$ & $€ 56.10$ & $€ 0.00$ & $€ I, 579.73$ \\
\hline IGla-100 bios. & $€ 193.30$ & $€ \mid 52.15$ & $€ 254.28$ & $€ 297.03$ & $€ 106.90$ & $€|30.7|$ & $€ 48.80$ & $€ 1,183.19$ \\
\hline Gliclazide & $€ \mid 30.76$ & $€ 76.08$ & $€ 95.80$ & $€ 208.85$ & $€ 77.31$ & $€ 82.47$ & $€ 48.80$ & $€ 720.07$ \\
\hline
\end{tabular}

Abbreviations: API, active pharmaceutical ingredient; SMBG, self-monitoring blood glucose; IHD, ischemic heart disease; HF, hospitalizations for heart failure; IGla-I00 bios, Insulin Glargine $100 \mathrm{U} / \mathrm{mL}$ Biosimilar. 
Table 6 Cost-Utility Analysis Results: Base Case Scenario

\begin{tabular}{|l|l|l|l|l|l|}
\hline API & Mean Cost per Patient & Mean QALYs per Patient & Delta Cost & Delta QALYs & ICER/QALYs \\
\hline Dulaglutide & $€ I, 579.73$ & 0.025 & & & \\
IGla-100 bios. & $€ I, 183.19$ & -0.015 & $€ 396.54$ & 0.040 & \\
Gliclazide & $€ 720.07$ & -0.015 & $€ 859.66$ & 0.040 & $€ 9913$ \\
\hline
\end{tabular}

Table 7 Summary of Cost-Utility Sensitivity Analysis Results

\begin{tabular}{|l|l|l|}
\hline Scenario & IGla-I 00 Bios. & Gliclazide \\
\hline Base case & $€ 9913$ & $€ 21,492$ \\
Costs $-20 \%$ & $€ 13,349$ & $€ 22,924$ \\
Costs $+20 \%$ & $€ 6478$ & $€ 20,059$ \\
Dulaglutide/lgla bios cost $-40 \%$ & Dominant & $€ 8722$ \\
Dulaglutide//gla bios cost $-45 \%$ & Dominant & $€ 7126$ \\
Dulaglutide/Igla bios cost $-50 \%$ & Dominant & $€ 5530$ \\
Efficacy - 20\% & $€ 15,854$ & $€ 28,299$ \\
Efficacy $+20 \%$ & $€ 5953$ & $€ 16,953$ \\
\hline
\end{tabular}

with three increasing discounts $(40 \%, 45 \%, 50 \%)$ applied to ex-factory price for non-generic drugs, in order to assess the cost-utility of dulaglutide adopting a discounted price closer to the real one. Sensitivity analysis findings are shown in Table 7 and Figure 2.

In all the scenarios, the cost-utility of dulaglutide has been demonstrated. Particularly, ICER has reported positive results in comparison with insulin glargine biosimilar. Focusing on the three scenarios with discounts applied to the ex-factory price of dulaglutide and insulin glargine, ICERs have shown that the once-weekly GLP-1 receptor agonist could be considered a dominant option compared to insulin glargine and it is characterized by an excellent cost-utility profile when compared to gliclazide. In Figure 3, direct costs of treatment in a scenario with $45 \%$ discount applied to dulaglutide and insulin glargine ex-factory price have been reported, clearly showing how much the drug purchase cost and complications costs affect the total and vary among different treatments.

With a dulaglutide purchase cost reduction of $45 \%$, chosen as an average percentage value in our simulation, the drug, due to its clinical benefits, despite a price still clearly higher than insulin glargine biosimilar, has reported a lower total cost, Figure 3.

\section{Discussion}

Our study aimed to evaluate the economic implications of once-weekly dulaglutide GLP-1 receptor agonist positive effects on cardiovascular outcomes and weight loss, versus gliclazide and basalization with insulin glargine based on recent clinical evidence. ${ }^{15,20}$ The cost-utility analysis, by defining the incremental cost-effectiveness ratio (ICER), has provided information on the additional resources that

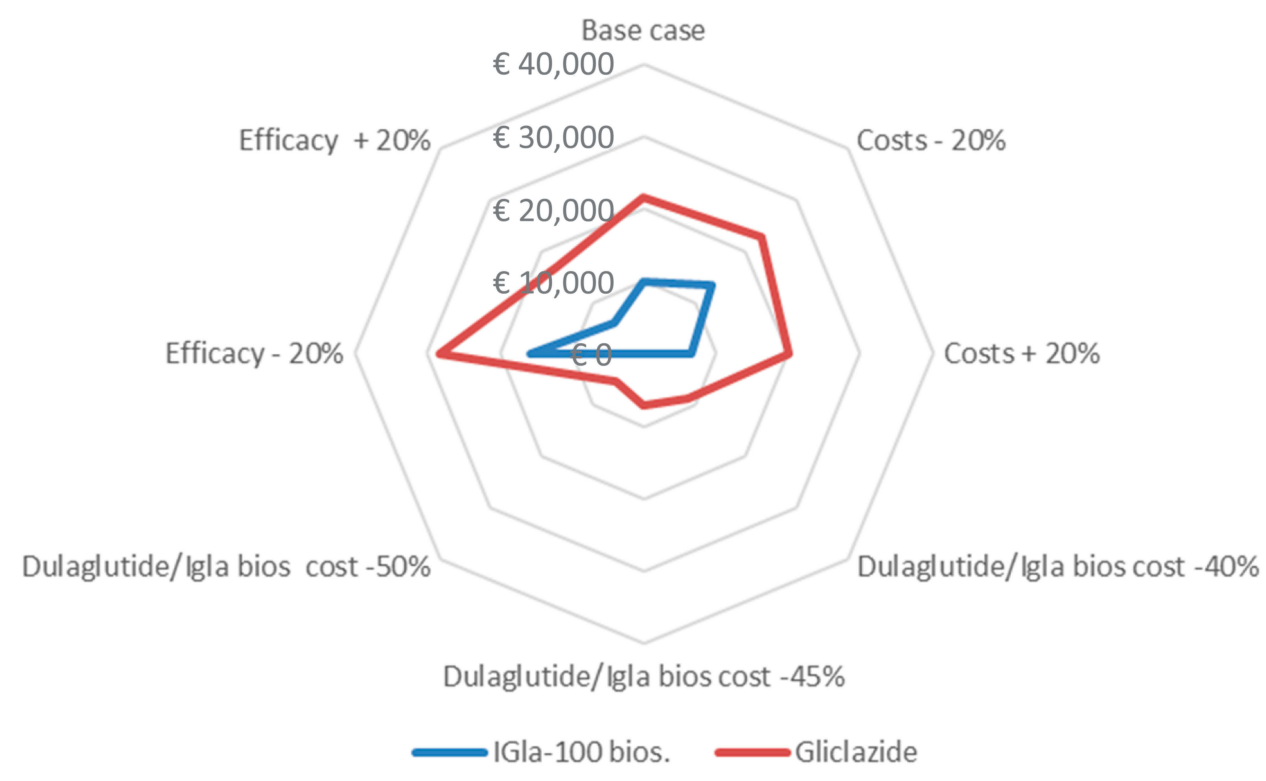

Figure 2 Findings of sensitivity analysis: cost per QALY gained. 


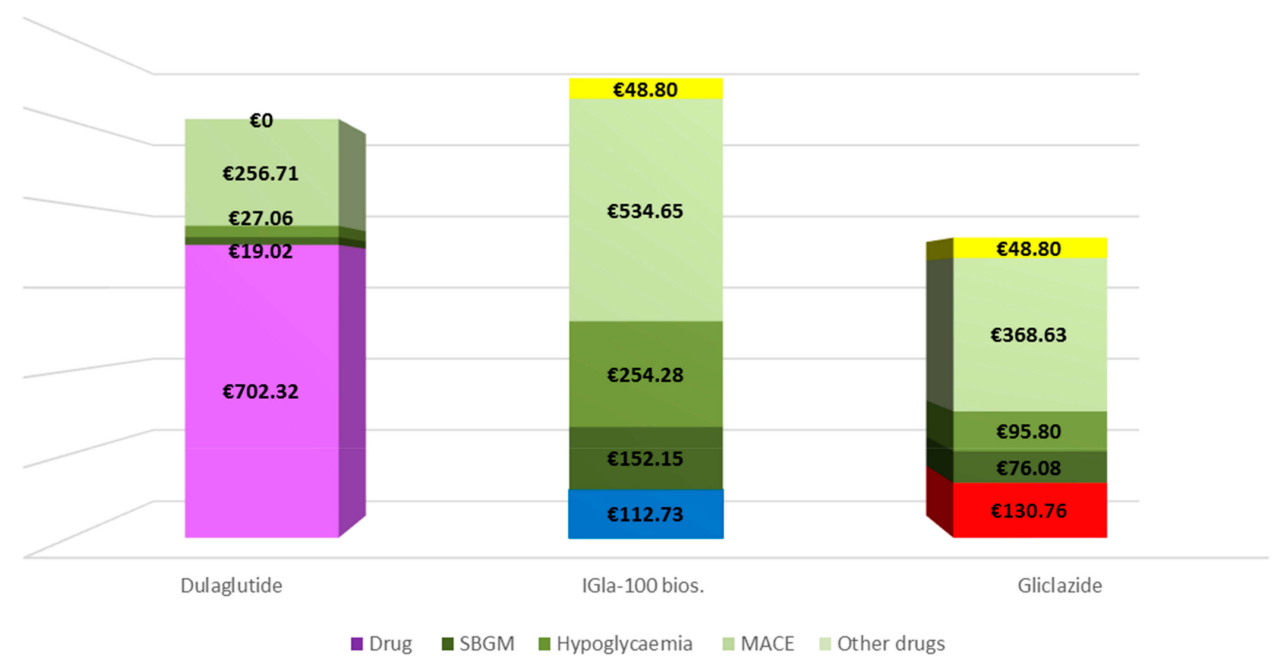

Figure 3 Direct costs for treatments: individual items for each treatment in a scenario with $45 \%$ discount applied to drug ex-factory price.

have to be used to achieve the additional benefit. In our analysis dulaglutide has found to be cost-effective when compared to gliclazide and insulin glargine biosimilar. In detail, our analysis has adopted total direct cost related to hypoglycemic treatments as cost measure, adopting the perspective of the Italian NHS, and the weight change impact on the quality of life as a parameter to calculate utilities scores. Total direct annual costs of treatments have been calculated adding cost items selected: drug cost, blood glucose self-monitoring, hypoglycaemic events cost, cardiovascular events cost (nonfatal acute myocardial infarction, stroke, and hospitalization for heart failure), and costs related to other drugs consumption. Final cost for each item has been obtained considering unit direct costs and frequency or rates. Although the difference between total direct costs of the compared treatments has been disadvantageous for dulaglutide, characterized by a purchase cost much higher than the others, which are now patent expired, lower costs associated with less need for monitoring, a better cardiovascular risk profile, a lower incidence of hypoglycaemic events and the favorable difference in terms of utility, have allowed to obtain positive ICER values. Indeed, our cost-utility findings have shown that once-weekly dulaglutide $1.5 \mathrm{mg}$ can improve qualityadjusted life-year (QALY) compared to insulin glargine and gliclazide as an add-on to metformin in T2DM patients inadequately controlled. Relative to insulin glargine and gliclazide, improvement in QALY has been accompanied by increases in cost, resulting in ICERs of $€ 9913$ and $€ 21,492$, respectively. In both comparisons ICER per QALY value has resulted below the accepted willingness to pay threshold, set in Italy at $€ 40,000,{ }^{25}$ although it has been particularly positive in comparison with insulin glargine biosimilar.

In a context of limited economic resources for NHSs, the higher acquisition cost of newer therapies has made health technology assessment (HTA) necessary to confirm the additional value of these therapies. To our knowledge, this is the first cost-utility analysis of dulaglutide versus gliclazide and insulin glargine in inadequately controlled on metformin monotherapy T2DM patients, aimed at defining the economic implications of these once-weekly GLP-1 agonist positive effects on cardiovascular risk and body weight, developed from the perspective of the Italian NHS. Results of our analysis have shown dulaglutide as a costeffectiveness therapeutic option from the perspective of the Italian NHS and are comparable with those of other recent economic evaluations conducted in other countries. ${ }^{42,43}$ In these studies, the cost-effectiveness of dulaglutide has been reported and in particular, the benefits on cardiovascular outcomes and weight loss are emphasized, without considering the additional benefits on adherence that can be derived from a weekly therapy instead of a daily one and from the minimally invasive dulaglutide administration system, not taken into consideration but to be evaluated in future studies. It is important to note that we have calculated drug cost based on ex-factory price for non-generic drugs, although it is not the real drug cost paid by health facilities in Italy, as a confidential discounted price is usually negotiated between manufacturers and Italian NHS. Thus, since at the basis of the gap between total direct costs in this analysis there is precisely the high purchase cost of dulaglutide, in order to 
evaluate the uncertainty around the real direct cost paid by hospitals, we have tested the effects of increasing discount rates applied to ex-factory price $(40 \%, 45 \%, 50 \%)$. The application of these discounts to dulaglutide and insulin glargine biosimilar ex-factory price has resulted in an extremely positive ICER compared to gliclazide, while it has made dulaglutide dominant in comparison with insulin glargine. Moreover, findings of our cost-utility analysis have resulted robust when tested in a sensitivity analysis that explored all key model parameters including both clinical and economic inputs, applying a $\pm 20 \%$ variation.

In literature, several studies have highlighted that the substantial cost of the newer drugs is offset by the benefits attainable on the reduction of complications, especially cardiovascular events and hypoglycaemia. This costutility has allowed us to not limit to the pure analysis of costs and provide a global assessment of dulaglutide therapy value, highlighting the economic benefits obtainable for the NHS, due to the enhancement of multiple clinical outcomes in diabetic patients and improvement of the quality of life.

As with all modeling analyses, this study is subject to limitations. First, as with any cost-effectiveness model, in the absence of a specific data pool to use, some key assumptions have been applied to extrapolate clinical trial data from studies. It should be specified that where data input for Italy has not been available, reliable international sources have been considered. So, due to the lack of suitable Italian data on the effect of drug-related body weight changes on health-related quality of life of T2DM patients, the utilities for the health states " $2 \mathrm{~kg}$ gain" and " $2 \mathrm{~kg}$ loss" adopted in the model have been derived from Riddestrale's Swedish study. ${ }^{24}$ To note that in our cost-utility analysis, we have estimated the utilities related solely to weight loss and this represents an element questionable. Moreover, the analysis essentially has considered the effects of hypoglycaemic and cardiovascular complications, while it has not included other types of comorbidities typically associated with diabetes and it has not evaluated the impact on adherence of the different frequencies and administration methods of the drugs in the analysis. Future long-term studies conducted in an Italian diabetic population are required to provide further evidence. It is a substantial challenge to identify accurate data on the various inputs required for modeling diabetes. Future analyses to develop robust diabetes-related utilities and disutilities specific to Italian population are likely to reduce current uncertainty. In any case, we have tried to use the best available data wherever possible and have tested results thoroughly by developing sensitivity analysis.

Our analysis has been carried out including only direct cost, to reflect the perspective of the Italian NHS. It can be considered a limit related to the choice of the analysis perspective; in economic evaluations conducted from the societal perspective, the inclusion of indirect costs is instead fundamental.

The addition of indirect costs to our analysis would make the benefit of dulaglutide even more evident, since it has shown clear positive outcomes on reduction of hypoglycaemic events and cardiovascular complications, which are characterized by a relevant impact on indirect costs. On the base of these considerations, we have developed an approximate calculation of the indirect costs associated with hypoglycaemic events (moderate and severe) and cardiovascular adverse events (IHD, stroke, HHF). Adding the direct costs to the indirect ones, we have obtained total costs, useful to express the social perspective and we have used these in the cost-utility analysis. Results of this additional scenario have shown ICERs extremely positive for dulaglutide. Specifically, in base case scenario ICER has been $€ 19,338$ when compared to gliclazide and $€ 2415$ when compared to insulin glargine. In this scenario, the gap between total annual cost, mainly determined by the purchase cost which represents the only cost component to the detriment of dulaglutide, is further reduced, given the high impact on indirect costs of diabetic complications, which are more frequent for gliclazide and insulin glargine. Another aspect to consider analyzing the real drug purchasing costs for NHS, it is the drug distribution cost. In Italy dulaglutide (Trulicity ${ }^{\circledR}$ ) was approved for reimbursement in February 2016 and it has been listed in the direct distribution formulary (Prontuario della Distribuzione Diretta ospedale -territorio, PHT) including reimbursable pharmaceuticals for patients that require specialized medical visits on a regular basis. ${ }^{44}$

In the future, dulaglutide and basal insulin may be dispensed through "Per Conto" distribution according to management chosen by each Region. In a cost analysis of different therapeutic options, it can, therefore, be useful to consider also distribution-related costs, in order to test the economic impact of "per Conto" distribution on final drugs cost.

Although drug costs are increasing, due to the progressive adoption of newer therapies, the greatest component of the economic burden of T2DM remains the treatment of diabetic complications, which can be reduced with effective 
management of the disease. In this context, the role of onceweekly dulaglutide GLP-1 receptor agonist stands out. Indeed, dulaglutide, based on its benefits on cardiovascular outcomes and weight loss, despite the high purchase cost, has been characterized as a cost-effective treatment and then an alternative therapeutic option compared to gliclazide and especially compared to insulin glargine.

\section{Conclusion}

In this cost-utility analysis, dulaglutide has shown to be a cost-effective treatment option from Italian healthcare system perspective as add-on therapy to metformin in patients with inadequately controlled type 2 diabetes mellitus. Study findings can provide stakeholders valuable evidence to support the adoption of this cost-effective second- or third-line therapy compared to gliclazide or basal insulin glargine. Dulaglutide cost-effectiveness has been particularly evident in the comparison with basal insulin glargine, indicating that, in patients who have treatment indication, this therapy may be preferred to basalization avoiding related complications and costs.

\section{Disclosure}

GLC, SDM, GMB, CM and MCV are employees of S.A.V. E. S.r.l and consultants for different pharmaceutical companies. ET and LCB are affiliated with Asl3. ET, in the last 3 years, has received consultancy and/or speaking fees and research grants from AstraZeneca, Boehringer Ingelheim, Bristol Myers Squibb, Eli Lilly, GSK, Janssen, Johnson \& Johnson, MSD Italy, Novo Nordisk, Sanofi and Takeda. The authors report no other conflicts of interest in this work.

\section{References}

1. ISTAT. National Institute of Statistics. Diabetes in Italy years 2000-2016. 2017. Available from: https://www.istat.it/en/files/2017/ 07/Report_Diabetes_En_def.pdf?title=Diabetes+in+Italy+-+24+Jul +2017+-+Full+text.pdf. Accessed January 30, 2018. English.

2. Osservatorio ARNO Diabete. Il profilo assistenziale della popolazione con diabete. Rapporto XXIII. 2015. Available from: http://osservator ioarno.cineca.org. Accessed December 7, 2015.

3. AMD. Le monografie degli annali. Focus su: Full Data Circle. Napoli: Idelson-Gnocchi; 2017.

4. Inzucchi SE, Bergenstal RM, Buse JB, et al. Management of hyperglycemia in type 2 diabetes: a patient-centered approach: position statement of the American Diabetes Association (ADA) and the European Association for the Study of Diabetes (EASD). Diabetes Care. 2012;35(6):1364-1379. doi:10.2337/dc12-0413

5. UK Prospective Diabetes Study (UKPDS) Group. Intensive blood glucose control with sulphonilureas or insulin compared with conventional treatment and risk of complications in patients with type 2 diabetes (UKPDS 33). Lancet. 1998;352(9131):837-853. doi:10.10 16/S0140-6736(98)07019-6
6. The Diabetes Control and Complications Trial Research Group. The effect of intensive treatment of diabetes on the development and progression of long-term complications in insulin-dependent diabetes mellitus. $N$ Engl J Med. 1993;329(14):977-986. doi:10.1056/NEJM199309303291401

7. Cryer PE. Hypoglycemia is the limiting factor in the management of diabetes. Diabetes Metab Res Rev. 1999;15(1):42-46. doi:10.1002/ (ISSN) $1520-7560$

8. Lorenzoni V, Baccetti F, Genovese S, et al. Cost-consequence analysis of sitagliptin versus sulfonylureas as add-on therapy for the treatment of diabetic patients in Italy. Clinicoecon Outcomes Res. 2017;9:699-710. doi:10.2147/CEOR

9. Iannazzo S, Mannucci E, Reifsnider O, et al. Cost-effectiveness analysis of empagliflozin in the treatment of patients with type 2 diabetes and established cardiovascular disease in Italy, based on the results of the EMPA-REG OUTCOME study. Farmeconomia Health Econ Ther Pathways. 2017;18(1):43-53. doi:10.7175/fe.v18i1.1332

10. Nauck M, Stöckmann F, Ebert R, Creutzfeldt W. Reduced incretin effect in type 2 (non-insulin-dependent) diabetes. Diabetologia. 1986;29(1):46-52. doi:10.1007/BF02427280

11. Vilsbøll T, Krarup T, Madsbad S, Holst JJ. Defective amplification of the late phase insulin response to glucose by GIP in obese type II diabetic patients. Diabetologia. 2002;45(8):1111-1119. doi:10.1007/ s00125-002-0878-6

12. Jendle J, Grunberger G, Blevins T, et al. Efficacy and safety of dulaglutide in the treatment of type 2 diabetes: a comprehensive review of the dulaglutide clinical data focusing on the AWARD phase 3 clinical trial program. Diabetes Metab Res Rev. 2016;32 (8):776-790. doi:10.1002/dmrr.v32.8

13. Zhang L, Zhang M, Zhang Y, Tong N. Efficacy and safety of dulaglutide in patients with type 2 diabetes: a meta-analysis and systematic review. Sci Rep. 2016;6(1):18904. doi:10.1038/srep18904

14. Tuttle KR, Lakshmanan MC, Rayner B, et al. Dulaglutide versus insulin glargine in patients with type 2 diabetes and moderate-tosevere chronic kidney disease (AWARD-7): a multicentre, open-label, randomised trial. Lancet Diabetes Endocrinol. 2018;6(8):605-617. doi:10.1016/S2213-8587(18)30104-9

15. Gerstein HC, Colhoun HM, Dagenais GR, et al. Dulaglutide and cardiovascular outcomes in type 2 diabetes (REWIND). A double blind, randomized placebo-controlled trial. lancet. 2019. doi:10.1016/ S0140-6736(19)31149-3

16. Dungan KM, Weitgasser R, Perez Manghi F, et al. A 24-week study to evaluate the efficacy and safety of once-weekly dulaglutide added on to glimepiride in type 2 diabetes (AWARD-8). Diabetes Obes Metab. 2016;18(5):475-482. doi:10.1111/dom.12634

17. Umpierrez G, Santiago Tofé Povedano F, Manghi P, et al. Efficacy and safety of dulaglutide monotherapy versus metformin in type 2 diabetes in a randomized controlled trial (AWARD-3). Diabetes Care. 2014;37(8):2168-2176. doi:10.2337/dc13-2759

18. Bethel MA, Patel RA, Merrill P, et al. Cardiovascular outcomes with glucagon-like peptide-1 receptor agonists in patients with type 2 diabetes: a meta-analysis. Lancet Diabetes Endocrinol. 2018;6 (2):105-113. doi:10.1016/S2213-8587(17)30412-6

19. ADA American Diabetes Association. Economic costs of diabetes in the U.S. in 2012. Diab Care. 2013;36(4):1033-1046. doi:10.2337/dc12-2625

20. O'Brien MJ, Karam SL, Wallia A, et al. Association of second-line antidiabetic medications with cardiovascular events among insured adults with type 2 diabetes. JAMA Network Open. 2018;1(8): e186125. doi:10.1001/jamanetworkopen.2018.6125

21. Gold MR, Siegel JE, Russell LB, Weinstein MC, editors. CostEffectiveness in Health and Medicine. New York: Oxford University Press; 1996.

22. Davies M, Storms F, Shutler S, Bianchi-Biscay M, Gomis R. For the Atlantus Study Group. improvement of glycemic control in subjects with poorly controlled type 2 diabetes. Comparison of two treatment algorithms using insulin glargine. Diabetes Care. 2005;28(6):12 82-1288. doi:10.2337/diacare.28.6.1282 
23. Khunti K, Caputo S, Damci T, et al. The safety and efficacy of adding once-daily insulin detemir to oral hypoglycaemic agents in patients with type 2 diabetes in a clinical practice setting in 10 countries. Diabetes Obes Metab. 2012;14(12):1129-1136. doi:10.1111/dom.20 12.14.issue-12

24. Ridderstråle M, Evans LM, Jensen HH, et al. Estimating the impact of changes in HbAlc, body weight and insulin injection regimen on health related quality-of-life: a time trade off study. Health Qual Life Outcomes. 2016;14(1):13. doi:10.1186/s12955-016-0411-0

25. Italian Health Economics Association Associazione Italiana di Economia Sanitaria - AIES. Proposta di linee guida per la valutazione economica degli interventi sanitari in Italia. [Italian guidelines proposal on how to conduct economic evaluation studies of health programs]. Pharmacoecon Ital Res Art. 2009;11(2):83-93. (). doi:10. 1007/BF03320660

26. Tunnel software Pharma Dati. Compendio Farmaceutico Farmadati Italia. [Pharma Data Italian Database]. Available from: https://www. farmadati.it/. Accessed March 21, 2020.

27. Associazione Medici Diabetologi (AMD). Societa Italiana di Diabetologia (SID) - Standard italiani per la cura del diabete mellito; 2016. Available from: www.standarditaliani.it. Accessed March 21, 2020.

28. Colombo GL, Torre E, Bruno G, Di Matteo S. Il costo della distribuzione diretta di sistemi di automonitoraggio della glicemia: un modello di analisi economica ClinicoEconomics. Ital Art Outcomes Res. 2014;9:13-24.

29. Liguria R. ASL n.3. Delibera per la fornitura di dispositivi per la determinazione della glicemia capillare. [Liguria region, Local health authority N.3. Decision for the provision of blood glucose monitoring devices].Available from: http://delibere.asl3.liguria.it/pdf/delibera zioni/2016/2016_700_1_77_Pubblico.pdf. Accessed March 21, 2020.

30. Jönsson L, Bolinder B, Lundkvist J. Cost of hypoglycemia in patients with type 2 diabetes in Sweden. Value in Health. 2006;9-3 (3):193-198. doi:10.1111/j.1524-4733.2006.00100.x

31. Leese GP, Wang G, Broomhall J, et al. Frequency of severe hypoglycemia requiring emergency treatment in type 1 and type 2 diabetes. Diabetes Care. 2003;26(4):1176-1180. doi:10.2337/diacare.26.4. 1176

32. Veronese G, Marchesini G, Forlani G, et al. Costs associated with emergency care and hospitalization for severe hypoglycaemia. Nutr Met CV Dis. 2016;26(4):345-351. doi:10.1016/j.numecd.2016.01.007

33. Eriksson JW, Bodegard J, Nathanson D, Thuresson M, Nystro"m T, Norhammar A. Sulphonylurea compared to DPP-4 inhibitors in combination with metformin carries increased risk of severe hypoglycemia, cardiovascular events, and all-cause mortality. Diabetes Res Clin Pract. 2016;117:39-47. doi:10.1016/j.diabres.2016.04.055

34. Lucioni C, Mazzi S, Rossi E, Rielli R, Calabria S, Maggioni AP, a nome dei partecipanti all'Osservatorio ARNO. Percorsi terapeutici e costi sanitari di pazienti ricoverati per un evento cardiovascolare in Italia. Glob Reg Health Technol Assess. 2016;3(2):80-91.
35. Bertoni AG, Hundley WG, Massing MW, Bonds DE, Burke GL, Goff DC Jr. Heart failure prevalence, incidence, and mortality in the elderly with diabetes. Diabetes Care. 2004;27(3):699-703. doi:10.2337/diacare.27.3.699

36. Corrao G, Ghirardi A, Ibrahim B, Merlino L, Maggioni AP. Burden of new hospitalization for heart failure: a population-based investigation from Italy. Eur J Heart Fail. 2014;16(7):729-736. doi:10.1002/ ejhf.2014.16.issue-7

37. Ferdinand KC, White WB, Calhoun DA, et al. Effects of the Once-weekly glucagon-like peptide-1 receptor agonist dulaglutide on ambulatory blood pressure and heart rate in patients with type 2 diabetes mellitus. Hypertension. 2014;64(4):731-737. doi:10.1161/ HYPERTENSIONAHA.114.03062

38. Gruppo Annali AMD, Annali AMD. Valutazione longitudinale 2004-2011 degli indicatori di qualità dell'assistenza al diabete di tipo 2 nelle regioni italiane. VIII ed. Torino; 2015. [AMD Annals group. AMD Annals. Long-term evaluation 2004-2011 of the quality indicators of type 2 diabetes care in the Italian regions. VIII ed. turin; 2015]. Available from: http://aemmedi.it/files/ANNALI-AMD/2014/ Annali\%20Regionali\%202014\%20web.pdf. Accessed March 08, 2018. Italian.

39. Brown RE, Gupta N, Aronson R. Effect of dapagliflozin on glycemic control, weight, and blood pressure in patients with type 2 diabetes attending a specialist endocrinology practice in Canada: a retrospective cohort analysis. Diabetes Technol Ther. 2017;19 (11):685-691. doi:10.1089/dia.2017.0134

40. Grandy S, Hashemi M, Langkilde AM, Parikh S, Sjöström CD. Changes in weight loss-related quality of life among type 2 diabetes mellitus patients treated with dapagliflozin. Diabetes Obes Metab. 2014;16(7):645-650. doi:10.1111/dom.12263

41. Hamann A, Garcia-Puig J, Paul G, et al. Comparison of fixed-dose rosiglitazone/metformin combination therapy with sulphonylurea plus metformin in overweight individuals with type 2 diabetes inadequately controlled on metformin alone. Exp Clin Endocrinol Diabetes. 2008;116(1):6-13. doi:10.1055/s-2007-984441

42. Pollock RF, Norrbacka K, Cameron C, et al. A cost-utility analysis of dulaglutide versus insulin glargine as third-line therapy for type 2 diabetes in Canada. J Comp Eff Res. 2019;8(4):229-240. doi:10.22 17/cer-2018-0073

43. Ishii H, Madin-Warburton M, Strizek A, et al. The cost-effectiveness of dulaglutide versus insulin glargine for the treatment of type 2 diabetes mellitus in Japan. J Med Econ. 2018;21(5):488-496. doi:10.1080/13696998.2018.1431918

44. Gazzetta Ufficiale Serie Generale n.45 del 24- 02-2016. Riclassificazione del medicinale per uso umano «Trulicity», ai sensi dell'articolo 8, comma 10, della legge 24 dicembre 1993, n. 537. (Determinan.29/2016). (16A01082) Available from: https://www.gaz zettaufficiale.it/eli/id/2016/02/24/16A01082/SG. Accessed March 20, 2020
ClinicoEconomics and Outcomes Research

\section{Publish your work in this journal}

ClinicoEconomics and Outcomes Research is an international, peerreviewed open-access journal focusing on Health Technology Assessment, Pharmacoeconomics and Outcomes Research in the areas of diagnosis, medical devices, and clinical, surgical and pharmacological intervention. The economic impact of health policy and health systems organization also constitute important areas of coverage. The manuscript management system is completely online and includes a very quick and fair peer-review system, which is all easy to use. Visit $\mathrm{http}: / / \mathrm{www}$.dovepress.com/testimonials.php to read real quotes from published authors. 